\title{
Longitudinal assessment of the impact of higher body mass index on cancer-related fatigue in patients with breast cancer receiving chemotherapy
}

\author{
Julia E. Inglis ${ }^{1}$, Michelle C. Janelsins ${ }^{1}$, Eva Culakova ${ }^{1}$, Karen M. Mustian ${ }^{1}$, Po-Ju Lin ${ }^{1}$, Ian \\ R. Kleckner ${ }^{1}$, Luke J. Peppone ${ }^{1}$ \\ ${ }^{1}$ Department of Surgery, University of Rochester Medical Center (URMC), 265 Crittenden Blvd, \\ CU 420658, Rochester, NY 14642, USA
}

\begin{abstract}
Purpose-To assess the impact of obesity on cancer-related fatigue (CRF) in patients with breast cancer, through a secondary analysis of a large, longitudinal, nationwide study of breast cancer patients beginning chemotherapy.

Methods-All patients $(N=565$; aged $53 \pm 10.6)$ with breast cancer completed the multidimensional fatigue symptom inventory and the symptom inventory to measure CRF symptoms at baseline, post-chemotherapy, and 6 months post-chemotherapy. Height and weight at baseline were used to categorize subjects based on body mass index (BMI): obese ( $\geq 30.0 \mathrm{~kg} / \mathrm{m}^{2}$; $n=294)$, overweight $\left(25.0-29.9 \mathrm{~kg} / \mathrm{m}^{2} ; n=146\right)$, and normal weight $\left(18.5-24.9 \mathrm{~kg} / \mathrm{m}^{2} ; n=125\right)$. Multivariate regression models evaluated the relationship of obesity level to CRF over time, controlling for age, menopausal status, race, Karnofsky Performance Status, cancer stage, radiation, and exercise status.
\end{abstract}

Results-At baseline, the obese had significantly higher CRF symptoms than the normal weight subjects for both the Multidimensional fatigue symptom inventory (MFSI) total (obese $=11.2 \mathrm{vs}$ normal weight $=6.3 ; p=0.03$ ) and Symptom Inventory (SI) (obese $=3.5$ vs normal weight $=2.9$; $p=0.03)$. Significantly higher SI fatigue scores persisted at post-chemotherapy for the obese (obese $=5.0 \mathrm{vs}$ normal weight $=4.4 ; p=0.02$ ). At 6 months post-chemotherapy, the obese patients still had significantly higher SI fatigue scores (obese $=3.5$ vs normal weight $=3.0 ; p=0.05$ ).

Conclusion-Obese patients suffered greater CRF from pre-chemotherapy through 6 months post-chemotherapy. Recommendations for weight loss or weight maintenance may impact CRF levels in obese breast cancer patients before and after chemotherapy.

Julia E. Inglis, Julia_Inglis@URMC.Rochester.edu.

Conflict of interest The authors declare that they have no conflict of interest.

Informed consent Informed consent was obtained from all individual participants included in the study.

Ethical approval Furthermore, the authors declare that the protocol herein described complies with the University of Rochester Medical Center and that they obtained institutional review board approval and have been performed in accordance with the ethical standards as laid down in the 1964 Declaration of Helsinki and its later amendments or comparable ethical standards.

Publisher's note Springer Nature remains neutral with regard to jurisdictional claims in published maps and institutional affiliations. 


\section{Keywords}

Cancer-related fatigue; Obesity; Breast cancer; Chemotherapy

\section{Introduction}

Over $50 \%$ of patients with breast cancer experience gain weight post-chemotherapy [1, 2]. In addition to the impact of treatment, there is already the increased prevalence of obesity in women postmenopause, so that women surviving breast cancer rarely return to their prediagnosis weight $[1,3,4]$. Obesity is also more prevalent in patients with breast cancer at the time of diagnosis, being a positive risk factor for disease development postmenopause [3-6].

Cancer-related fatigue (CRF) is experienced by up to $90 \%$ of patients with breast cancer and is considered the most persistent and distressing physical impairment post-treatment [7-9]. CRF differs from other types of fatigue in that its development is unrelated to physical overexertion or recent sleeplessness and may persist for years in breast cancer survivors with little improvement [10,11]. Quality of life (QOL) may also be impacted, so that interpersonal relationships, employment, and work productivity fall into decline [8, 11]. Inflammatory cytokine production and disruption of metabolic processes resulting from cancer and cancer treatment contribute to CRF [12,13]. Notably, as fat tissue increases in obese patients with breast cancer, there is increased dysfunctional secretion of endocrine factors and inflammatory molecules present. This process is known as the "adipokine hypothesis," in which adipose cells secrete pro-inflammatory cytokines, much like an endocrine organ $[14,15]$. Considering that inflammation promotes CRF and that obese patients have increased chronic low-grade inflammation, it is reasonable to tie obesity in breast cancer patients to CRF and assume CRF may persist longer in this population [16, 17].

In addition, breast cancer survivors often gain weight as a side effect of chemotherapy or hormonal therapy, while simultaneously losing or failing to gain adequate lean tissue, causing the development of the condition termed sarcopenic obesity [18, 19]. This combined condition is characterized by muscle depletion or inadequate levels of lean mass, producing further weakness in the patient with cancer [20]. Sarcopenic obesity in breast cancer survivors could further exacerbate CRF symptoms [21,22]. Therefore, the impact of CRF in obese patients and survivors may be an area of concern with the increase in obese patients worldwide, especially in patients with breast cancer [23,24]. The aim of this study was to evaluate the impact of obesity on CRF in breast cancer patients by means of a secondary analysis of a prospective study of breast cancer patients undergoing chemotherapy [25].

\section{Methods}

\section{Study population}

Data were collected from a prospective, longitudinal nationwide study examining chemotherapy and cognitive function in patients with a diagnosis of invasive breast cancer (stages I to IIIC) [25]. Patients were treated at community oncology clinics affiliated with 
the University of Rochester Cancer Control (URCC) National Cancer Institute Community Oncology Research Program (NCORP) Research Base. This secondary analysis evaluated 565 breast cancer patients from pre-chemotherapy until 6 months post-chemotherapy.

Outcomes were appraised at three points: within 7 days before chemotherapy (prechemotherapy, baseline), within 4 weeks after chemotherapy completion (postchemotherapy), and 6 months post-chemotherapy (6 months follow-up). Institutional review boards at the University of Rochester and each of the 22 NCORP sites approved the study prior to participant enrollment. A complete description of methods was listed previously [25].

\section{Measures}

In this analysis, participants were categorized by obesity status based on body mass index (BMI) at baseline: obese ( $\left.\geq 30.0 \mathrm{~kg} / \mathrm{m}^{2} ; n=294\right)$, overweight $\left(25.0-29.9 \mathrm{~kg} / \mathrm{m}^{2} ; n=146\right.$ ), and normal weight $\left(18.5-24.9 \mathrm{~kg} / \mathrm{m}^{2} ; n=125\right)$. Height and weight were obtained from the clinical record via clinical exam. Karnofsky performance status (KPS), menopausal status, radiation history, and cancer stage were obtained from medical records. Race and exercise status were self-reported where exercise status was based on whether or not participants stated that they exercised weekly on a regular basis over the past 6 months (see Table 1).

Serum albumin levels were measured to evaluate potential malnutrition among groups, although albumin levels may also be influenced by inflammation and stress from cancer and cancer treatment and other chronic conditions [26-28]. Albumin levels were only available at baseline and post-chemotherapy (see Table 2). Symptoms related to CRF were evaluated by the multidimensional fatigue symptom inventory-short form (MFSI-SF) and a CRF question from the Symptom Inventory (SI) questionnaire at all three assessment points. Both the MFSI and SI are reliable and validated in cancer patients and survivors [29, 30].

\section{Statistical analyses}

Distribution of baseline characteristics was evaluated, and mean (standard deviation) and $n$ $(\%)$ were reported for continuous and categorical measures, respectively. Data were analyzed at baseline, post-chemotherapy, and 6 months post-chemotherapy to evaluate the relationship of baseline obesity level to CRF over time. Missing data were analyzed to ensure they were missing completely at random, across all groups of participants. If data in a variable appeared to not be missing at random, and there were significant missing data in just one group, such as the "obese" group, then that variable would be further evaluated to understand if there was a bias in the model or data collection method. There were 11 participants with missing BMI values at baseline due to having no weight records at baseline, making it impossible to calculate their BMI. Since this is a small proportion of participants (2\%) and the paper is about obesity, we excluded these participants from the analysis. More than $50 \%$ of the data for participants' weight were missing for the postchemo and the 6 months follow-up assessments; therefore, analysis of change in weight over time was not feasible. In general, in the multivariate analysis, if $<5 \%$ of data were missing for any variable, we included the variable in the model. In the case with albumin, however, $\sim 25 \%$ of data was missing for all participants at both baseline and post-chemotherapy. However, this is noted later in the paper. 
Differences in CRF levels between groups at each time point were assessed using multivariate regression models. Statistical models were adjusted for age, menopausal status, race, KPS, cancer stage, radiation, and exercise status. To confirm the results over time, additional analysis was conducted using linear mixed modeling (LMM) incorporating data from all three time points as repeated measures per participant. A $p$ value $<0.05$ was considered significant. All calculations were conducted in SAS 9.4 (SAS Institute, Cary, NC).

\section{Results}

This analysis examined CRF symptoms in 565 female patients with breast cancer (mean age $53 \pm 10.6$ years). For this analysis, 11 participants from the original study were excluded due to extensive missing data related to body weight (BMI) and CRF. At baseline, obese and overweight participants were more likely to be older and postmenopausal (see Table 1). Nearly half did not exercise over the past 6 months across BMI categories. There were no differences in race, KPS, exercise status, cancer stage, radiation history, or other treatment between groups. Serum albumin levels were assessed at baseline and post-chemotherapy, with mean values for all groups at approximately $4.0 \mathrm{~g} / \mathrm{dL}$, although there was missing data for albumin for $25 \%$ of participants. At post-chemotherapy, there was a decrease in albumin levels for the obese group with a trend toward significance $(p=0.052)$ among the obese $(3.9$ $\mathrm{g} / \mathrm{dL}$ ) and the other groups $(4.0 \mathrm{~g} / \mathrm{dL})$ (see Table 2$)$.

\section{Obesity and cancer-related fatigue}

All results for CRF are presented as adjusted means. Obesity status throughout the study was categorized based on baseline BMI calculations. Obese patients with cancer consistently presented with the highest scores for CRF symptoms. The obese had greater fatigue at baseline than normal weight participants, with all three groups experiencing an increase in fatigue post-chemotherapy treatment and a decrease in fatigue levels 6 months follow-up post-chemotherapy, where they returned to almost baseline levels (see Figs. 1 and 2). The obese group consistently presented with significantly greater fatigue than the normal weight participants based on the SI fatigue question at baseline and post-chemotherapy and at 6 months post-chemotherapy ( $p<0.05$; see Fig. 1). These results were confirmed in the linear mixed model (LMM) analysis, showing significant changes over time. Generally, a score of 4 or higher on the 11-point SI fatigue question based on fatigue level over the past week indicates CRF in cancer patients and survivors [30]. Mean scores reached 4 and greater at post-chemotherapy in this analysis. Furthermore, throughout the study, the obese patients had higher scores than the normal weight participants based on the MFSI total and MFSI general, although the difference was only significant at the baseline (see Fig. 2). MFSI general may suggest poorer QOL in their experience with cancer and cancer treatment. All other MFSI subscales: physical, emotional, mental and vigor scales were also evaluated among groups without an increased score in the obese (see Table 2).

\section{Discussion}

This longitudinal prospective study demonstrated a pattern of greater CRF in the obese patients with breast cancer than normal weight patients over time, from baseline through 6 
months follow-up post-chemotherapy. Obesity status was based on baseline BMI where more than half of the participants were obese, which is common in patients with breast cancer. MFSI and SI questionnaires, which were used to assess CRF symptoms, are both validated and reliable in other studies examining CRF in cancer patients and survivors [29, 31, 30]. Based on the SI and MFSI, CRF symptoms increased from baseline to postchemotherapy and then decreased somewhat again at 6 months follow-up postchemotherapy. Previous research found that sarcopenic patients have lower levels of serum albumin [32]. In this study, serum albumin levels were normal across all groups, although decreased slightly in the obese group post-chemotherapy. Few other studies have evaluated the relationship of obesity with CRF levels in breast cancer patients longitudinally.

Obese patients experience chronic low-grade inflammation which may further contribute to the disease. The "adipokine hypothesis" asserts that white adipose cells may function as an endocrine organ, secreting hormones that in an auto and paracrine fashion which further contributes to chronic disease [15]. Expanding fat stores in the obese also can lead to dysfunctional secretion of endocrine factors and inflammatory molecules that potentially impact obese breast cancer patients, both preand post-treatment [15, 33]. Furthermore, some studies even link the type of gut microbiota in the obese patient to increased proinflammatory cytokine production and secretion [34]. Pro-inflammatory cytokines including interleukin (IL)-1, IL-6, and TNF- $a$ are found in the cancer tumor microenvironment and are also believed to play a mechanistic role in CRF development in breast cancer patients [35]. What the role of obesity and chronic low-grade inflammation plays in CRF development in this population is yet to be elucidated, but findings from this analysis suggest that the obese patient suffers a greater burden of fatigue. Future research is needed to identify which inflammatory molecules are most likely to increase CRF in obese breast cancer patients.

This study supports previous research on CRF in patients with breast cancer. In a study assessing CRF in 337 breast cancer survivors 6 to 42 months post-treatment, those with CRF had higher BMIs and were more likely to be obese at baseline [36]. Other research in breast cancer survivors has also correlated higher BMI with CRF [37-39]. However, the study by Taylor et al. also recognized that greater CRF levels were partly explained by lower activity levels in the obese group [39].

Previous studies have also demonstrated that many times, symptoms present at prechemotherapy in cancer patients often become worse post-treatment [40]. In this study, there was increased fatigue with chemotherapy treatment, with subsequent improvement in CRF in all groups 6 months post-treatment, with a persistent difference between obese and nonobese patients. Obesity may play a role in worsening CRF over the course of treatment as well as following chemotherapy, suggesting that weight loss therapy might be warranted in this population pre-chemotherapy [41]. Furthermore, the obese and overweight patients were more likely to be post-menopausal. Weight loss is more difficult postmenopause, due to decreased metabolic rate and lean mass and hormonal changes that lead to increased body fat $[42,43]$. Previous studies suggest that this population requires a combined treatment approach that includes increased physical activity, dietary changes, and other behavioral modifications for weight loss interventions to be effective [41, 44, 45]. Currently, most 
research on treatment for CRF in breast cancer patients focuses on the impact of exercise or physical activity [46-48]. Further research is needed on the impact of dietary changes, diet combined with exercise interventions, and weight loss on CRF in breast cancer patients and survivors.

Strengths of this study include a large, homogenous, nationwide sample size of patients with breast cancer.

Limitations involve the preliminary nature of this analysis. To evaluate the impact of obesity on fatigue in cancer patients in the future, it would be prudent to also measure levels of proinflammatory cytokines in relation to obesity and CRF. This study did not control for chemotherapy dose, sleep quality, or for sociological data such as income level.

\section{Conclusion}

In this study, obese patients with breast cancer presented with higher levels of CRF than normal weight patients pre-chemotherapy, over the course of chemotherapy and 6 months post-chemotherapy. Obesity levels at pre-chemotherapy may impact CRF throughout treatment, as well as the severity and duration of CRF post-chemotherapy in patients with breast cancer. Most interventions in breast cancer survivors to reduce CRF focus on exercise and physical activity. Postmenopausal women with breast cancer struggle more with weight loss and obesity. More research is needed on weight loss interventions as well as dietary changes, possibly combined with exercise, to address CRF in obese patients with breast cancer.

\section{Acknowledgments}

The authors would like to thank all study participants from NCORP sites involved in this research.

Funding information This study was funded by grants U10CA037420, UG1 CA189961, DP2 CA195765, R25 CA1026185, and K07CA221931 through the National Cancer Institute in the National Institute of Health.

\section{Abbreviations}

CRF

BMI Body mass index

QOL

SI

MFSI-SF

KPS

Quality of life
Cancer-related fatigue

Symptom inventory

Multidimensional fatigue symptom inventory-short form

Karnofsky Performance Status

\section{References}

1. Goodwin PJ, Ennis M, Pritchard KI, McCready D, Koo J, Sidlofsky S, Trudeau M, Hood N, Redwood S (1999) Adjuvant treatment and onset of menopause predict weight gain after breast cancer diagnosis. J Clin Oncol Off J Am Soc Clin Oncol 17(1):120-129. 10.1200/jco.1999.17.1.120 
2. Cantarero-Villanueva I, Galiano-Castillo N, Fernandez-Lao C, Diaz-Rodriguez L, Fernandez-Perez AM, Sanchez MJ, Arroyo-Morales M (2015) The influence of body mass index on survival in breast cancer patients. Clin Breast Cancer 15(2):e117-e123. 10.1016/j.clbc.2014.11.006 [PubMed: 25499695]

3. Neuhouser ML, Aragaki AK, Prentice RL, Manson JE, Chlebowski R, Carty CL, Ochs-Balcom HM, Thomson CA, Caan BJ, Tinker LF, Urrutia RP, Knudtson J, Anderson GL (2015) Overweight, obesity, and postmenopausal invasive breast cancer risk: a secondary analysis of the women's health initiative randomized clinical trials. JAMA Oncol 1(5):611-621. 10.1001/jamaoncol.2015.1546 [PubMed: 26182172]

4. Yerushalmi R, Dong B, Chapman JW, Goss PE, Pollak MN, Burnell MJ, Levine MN, Bramwell VHC, Pritchard KI, Whelan TJ, Ingle JN, Shepherd LE, Parulekar WR, Han L, Ding K, Gelmon KA (2017) Impact of baseline BMI and weight change in CCTG adjuvant breast cancer trials. Ann Oncol 28(7):1560-1568. 10.1093/annonc/mdx152 [PubMed: 28379421]

5. Ford ME, Magwood G, Brown ET, Cannady K, Gregoski M, Knight KD, Peterson LL, Kramer R, Evans-Knowell A, Turner DP (2017) Disparities in obesity, physical activity rates, and breast cancer survival. Adv Cancer Res 133:23-50. 10.1016/bs.acr.2016.08.002 [PubMed: 28052820]

6. Picon-Ruiz M, Morata-Tarifa C, Valle-Goffin JJ, Friedman ER, Slingerland JM (2017) Obesity and adverse breast cancer risk and outcome: mechanistic insights and strategies for intervention. CA Cancer J Clin 67(5):378-397. 10.3322/caac.21405 [PubMed: 28763097]

7. Hall DL, Mishel MH, Germino BB (2014) Living with cancer-related uncertainty: associations with fatigue, insomnia, and affect in younger breast cancer survivors. Support Care Cancer 22(9): 24892495. 10.1007/s00520-014-2243-y [PubMed: 24728586]

8. Peoples AR, Roscoe JA, Block RC, Heckler CE, Ryan JL, Mustian KM, Janelsins MC, Peppone LJ, Moore DF Jr, Coles C, Hoelzer KL, Morrow GR, Dozier AM (2017) Nausea and disturbed sleep as predictors of cancer-related fatigue in breast cancer patients: a multicenter NCORP study. Support Care Cancer 25(4):1271-1278. 10.1007/s00520-016-3520-8 [PubMed: 27995318]

9. Bower JE (2014) Cancer-related fatigue-mechanisms, risk factors, and treatments. Nat Rev Clin Oncol 11(10):597-609. 10.1038/nrclinonc.2014.127 [PubMed: 25113839]

10. Crosswell AD, Lockwood KG, Ganz PA, Bower JE (2014) Low heart rate variability and cancerrelated fatigue in breast cancer survivors. Psychoneuroendocrinology 45:58-66. 10.1016/ j.psyneuen.2014.03.011 [PubMed: 24845177]

11. Barsevick AM, Irwin MR, Hinds P, Miller A, Berger A, Jacobsen P, Ancoli-Israel S, Reeve BB, Mustian K, O’Mara A, Lai JS, Fisch M, Cella D (2013) Recommendations for high-priority research on cancer-related fatigue in children and adults. J Natl Cancer Inst 105(19):1432-1440. 10.1093/jnci/djt242 [PubMed: 24047960]

12. Bower JE, Lamkin DM (2013) Inflammation and cancer-related fatigue: mechanisms, contributing factors, and treatment implications. Brain Behav Immun 30(Suppl):S48-S57. 10.1016/ j.bbi.2012.06.011 [PubMed: 22776268]

13. Kuhl T, Behrens S, Jung AY, Obi N, Thone K, Schmidt ME, Becher H, Chang-Claude J (2018) Validation of inflammatory genetic variants associated with long-term cancer related fatigue in a large breast cancer cohort. Brain Behav Immun. 10.1016/j.bbi.2018.05.009

14. Izaola O, de Luis D, Sajoux I, Domingo JC, Vidal M (2015) [Inflammation and obesity (lipoinflammation)]. Nutr Hosp 31(6): 2352-2358. 10.3305/nh.2015.31.6.8829 [PubMed: 26040339]

15. Chadt A, Scherneck S, Joost HG, Al-Hasani H (2000) Molecular links between obesity and diabetes: "diabesity” In: De Groot LJ, Chrousos G, Dungan K et al. (eds). Endotext. MD Text.com, Inc., South Dartmouth (MA)

16. Schmitz KH, Neuhouser ML, Agurs-Collins T, Zanetti KA, Cadmus-Bertram L, Dean LT, Drake BF (2013) Impact of obesity on cancer survivorship and the potential relevance of race and ethnicity. J Natl Cancer Inst 105(18):1344-1354. 10.1093/jnci/djt223 [PubMed: 23990667]

17. Meeske K, Smith AW, Alfano CM, McGregor BA, McTiernan A, Baumgartner KB, Malone KE, Reeve BB, Ballard-Barbash R, Bernstein L (2007) Fatigue in breast cancer survivors two to five years post diagnosis: a HEAL study report. Qual Life Res Int J Qual Life Asp Treat Care Rehab 16(6):947-960. 10.1007/s11136-007-9215-3 
18. Demark-Wahnefried W, Kenyon AJ, Eberle P, Skye A, Kraus WE (2002) Preventing sarcopenic obesity among breast cancer patients who receive adjuvant chemotherapy: results of a feasibility study. Clin Exerc Physiol 4(1):44-49 [PubMed: 16946801]

19. Deluche E, Leobon S, Desport JC, Venat-Bouvet L, Usseglio J, Tubiana-Mathieu N (2018) Impact of body composition on outcome in patients with early breast cancer. Support Care Cancer 26(3):861-868. 10.1007/s00520-017-3902-6 [PubMed: 28948392]

20. Martin L, Birdsell L, Macdonald N, Reiman T, Clandinin MT, McCargar LJ, Murphy R, Ghosh S, Sawyer MB, Baracos VE (2013) Cancer cachexia in the age of obesity: skeletal muscle depletion is a powerful prognostic factor, independent of body mass index. J Clin Oncol Off J Am Soc Clin Oncol 31(12):1539-1547. 10.1200/jco.2012.45.2722

21. Kilgour RD, Vigano A, Trutschnigg B, Hornby L, Lucar E, Bacon SL, Morais JA (2010) Cancerrelated fatigue: the impact of skeletal muscle mass and strength in patients with advanced cancer. J Cachexia Sarcopenia Muscle 1(2):177-185. 10.1007/s13539-010-0016-0 [PubMed: 21475694]

22. Platt A, Gross J, Davis MP (2015) Force perception, effort, strength, and dynamic muscle function in cancer-related fatigue: a pilot study. Am J Hosp Palliat Care 32(6):577-580. 10.1177/1049909114528746 [PubMed: 24707008]

23. Jeronimo AF, Freitas AG, Weller M (2017) Risk factors of breast cancer and knowledge about the disease: an integrative revision of Latin American studies. Cienc Saude Colet 22(1):135-149. 10.1590/1413-81232017221.09272015

24. Nagrani R, Mhatre S, Rajaraman P, Soerjomataram I, Boffetta P, Gupta S, Parmar V, Badwe R, Dikshit R (2016) Central obesity increases risk of breast cancer irrespective of menopausal and hormonal receptor status in women of South Asian ethnicity. Eur J Cancer 66:153-161. 10.1016/ j.ejca.2016.07.022 [PubMed: 27573429]

25. Janelsins MC, Heckler CE, Peppone LJ, Kamen C, Mustian KM, Mohile SG, Magnuson A, Kleckner IR, Guido JJ, Young KL, Conlin AK, Weiselberg LR, Mitchell JW, Ambrosone CA, Ahles TA, Morrow GR (2017) Cognitive complaints in survivors of breast cancer after chemotherapy compared with age-matched controls: an analysis from a nationwide, multicenter, prospective longitudinal study. J Clin Oncol Off J Am Soc Clin Oncol 35(5):506-514. 10.1200/ jco.2016.68.5826

26. Aldebeyan S, Nooh A, Aoude A, Weber MH, Harvey EJ (2017) Hypoalbuminaemia-a marker of malnutrition and predictor of postoperative complications and mortality after hip fractures. Injury 48(2):436-440. 10.1016/j.injury.2016.12.016 [PubMed: 28040258]

27. Lim WS, Roh JL, Kim SB, Choi SH, Nam SY, Kim SY (2017) Pretreatment albumin level predicts survival in head and neck squamous cell carcinoma. Laryngoscope 127(12):E437-e442. 10.1002/ lary.26691 [PubMed: 28561532]

28. Tada T, Kumada T, Toyoda H, Yasuda S, Koyabu T, Nakashima M (2019) Impact of branchedchain amino acid granule therapy in patients with hepatocellular carcinoma who have normal albumin levels and low branched-chain amino acid to tyrosine ratios. Nutr Cancer:1-10. 10.1080/01635581.2019.1597905

29. Stein KD, Jacobsen PB, Blanchard CM, Thors C (2004) Further validation of the multidimensional fatigue symptom inventory-short form. J Pain Symptom Manag 27(1):14-23

30. Jones D, Zhao F, Fisch MJ, Wagner LI, Patrick-Miller LJ, Cleeland CS, Mendoza TR (2014) The validity and utility of the MD Anderson symptom inventory in patients with prostate cancer: evidence from the Symptom Outcomes and Practice Patterns (SOAPP) data from the Eastern Cooperative Oncology Group. Clin Genitourin Cancer 12(1):41-49. 10.1016/j.clgc.2013.07.003 [PubMed: 24126238]

31. Donovan KA, Stein KD, Lee M, Leach CR, Ilozumba O, Jacobsen PB (2015) Systematic review of the multidimensional fatigue symptom inventory-short form. Support Care Cancer 23(1):191-212. 10.1007/s00520-014-2389-7 [PubMed: 25142703]

32. Can B, Kara O, Kizilarslanoglu MC, Arik G, Aycicek GS, Sumer F, Civelek R, Demirtas C, Ulger Z (2017) Serum markers of inflammation and oxidative stress in sarcopenia. Aging Clin Exp Res 29(4):745-752. 10.1007/s40520-016-0626-2 [PubMed: 27571781]

33. Karam BS, Chavez-Moreno A, Koh W, Akar JG, Akar FG (2017) Oxidative stress and inflammation as central mediators of atrial fibrillation in obesity and diabetes. Cardiovasc Diabetol 16(1): 120 10.1186/s12933-017-0604-9 [PubMed: 28962617] 
34. Saad MJ, Santos A, Prada PO (2016) Linking gut microbiota and inflammation to obesity and insulin resistance. Physiology (Bethesda, Md) 31(4):283-293. 10.1152/physiol.00041.2015

35. Weber D, O'Brien K (2017) Cancer and cancer-related fatigue and the interrelationships with depression, stress, and inflammation. J Evid Based Complementary Altern Med 22(3):502-512. 10.1177/2156587216676122 [PubMed: 30208733]

36. Andrykowski MA, Donovan KA, Laronga C, Jacobsen PB (2010) Prevalence, predictors, and characteristics of off-treatment fatigue in breast cancer survivors. Cancer 116(24):5740-5748. 10.1002/cncr.25294 [PubMed: 20734399]

37. Gerber LH, Stout N, McGarvey C, Soballe P, Shieh CY, Diao G, Springer BA, Pfalzer LA (2011) Factors predicting clinically significant fatigue in women following treatment for primary breast cancer. Support Care Cancer 19(10):1581-1591. 10.1007/s00520-010-0986-7 [PubMed: 20835835]

38. Reinertsen KV, Cvancarova M, Loge JH, Edvardsen H, Wist E, Fossa SD (2010) Predictors and course of chronic fatigue in long-term breast cancer survivors. J Cancer Surviv 4(4):405-414. 10.1007/s11764-010-0145-7 [PubMed: 20862614]

39. Taylor DL, Nichols JF, Pakiz B, Bardwell WA, Flatt SW, Rock CL (2010) Relationships between cardiorespiratory fitness, physical activity, and psychosocial variables in overweight and obese breast cancer survivors. Int J Behav Med 17(4):264-270. 10.1007/s12529-010-9076-y [PubMed: 20177847]

40. Ancoli-Israel S, Liu L, Rissling M, Natarajan L, Neikrug AB, Palmer BW, Mills PJ, Parker BA, Sadler GR, Maglione J (2014) Sleep, fatigue, depression, and circadian activity rhythms in women with breast cancer before and after treatment: a 1-year longitudinal study. Support Care Cancer 22(9):2535-2545. 10.1007/s00520-014-2204-5 [PubMed: 24733634]

41. Reeves MM, Terranova CO, Eakin EG, Demark-Wahnefried W (2014) Weight loss intervention trials in women with breast cancer: a systematic review. Obes Rev 15(9):749-768. 10.1111/ obr.12190 [PubMed: 24891269]

42. Demark-Wahnefried W, Campbell KL, Hayes SC (2012) Weight management and its role in breast cancer rehabilitation. Cancer 118(8 Suppl):2277-2287. 10.1002/cncr.27466 [PubMed: 22488702]

43. Gravena AA, Brischiliari SC, Lopes TC, Agnolo CM, Carvalho MD, Pelloso SM (2013) Excess weight and abdominal obesity in postmenopausal Brazilian women: a population-based study. BMC Womens Health 13:46 10.1186/1472-6874-13-46 [PubMed: 24228934]

44. Mefferd K, Nichols JF, Pakiz B, Rock CL (2007) A cognitive behavioral therapy intervention to promote weight loss improves body composition and blood lipid profiles among overweight breast cancer survivors. Breast Cancer Res Treat 104(2):145-152. 10.1007/s10549-006-9410-x [PubMed: 17058023]

45. Campbell KL, Van Patten CL, Neil SE, Kirkham AA, Gotay CC, Gelmon KA, McKenzie DC (2012) Feasibility of a lifestyle intervention on body weight and serum biomarkers in breast cancer survivors with overweight and obesity. J Acad Nutr Diet 112(4): 559-567. 10.1016/ j.jada.2011.10.022 [PubMed: 22709706]

46. Meneses-Echavez JF, Gonzalez-Jimenez E, Ramirez-Velez R (2015) Effects of supervised exercise on cancer-related fatigue in breast cancer survivors: a systematic review and meta-analysis. BMC Cancer 15:77 10.1186/s12885-015-1069-4 [PubMed: 25885168]

47. Lipsett A, Barrett S, Haruna F, Mustian K, O’Donovan A (2017) The impact of exercise during adjuvant radiotherapy for breast cancer on fatigue and quality of life: a systematic review and meta-analysis. Breast 32:144-155. 10.1016/j.breast.2017.02.002 [PubMed: 28189100]

48. Lin PJ, Peppone LJ, Janelsins MC, Mohile SG, Kamen CS, Kleckner IR, Fung C, Asare M, Cole CL, Culakova E, Mustian KM (2018) Yoga for the management of cancer treatment-related toxicities. Curr Oncol Rep 20(1):5 10.1007/s11912-018-0657-2 [PubMed: 29388071] 
6

SI Fatigue Scores

$$
p=.018^{*}
$$

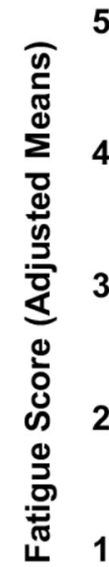

0

$$
p=.028 *
$$

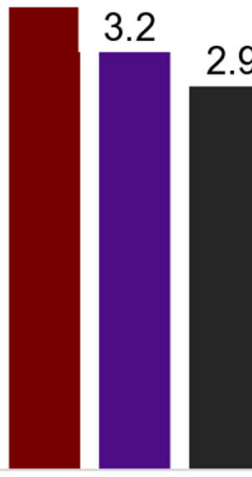

Baseline
$5.0 \quad 4.9$

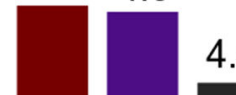

\section{4}

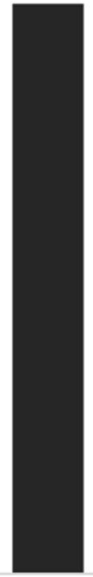
$p=.046^{*}$

- Obese

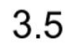

3.4

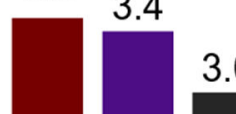

3.0

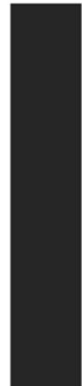

- Normal

Fig. 1.

SI Fatigue scores baseline, post-chemotherapy, and 6 months follow-up post-chemotherapy based on baseline obesity categorization. $* p$ value obese vs. normal weight participants. SI fatigue, Symptom Inventory fatigue. Means adjusted for age, menopausal status, race, Karnofsky Performance Status, cancer stage, radiation, and exercise status 

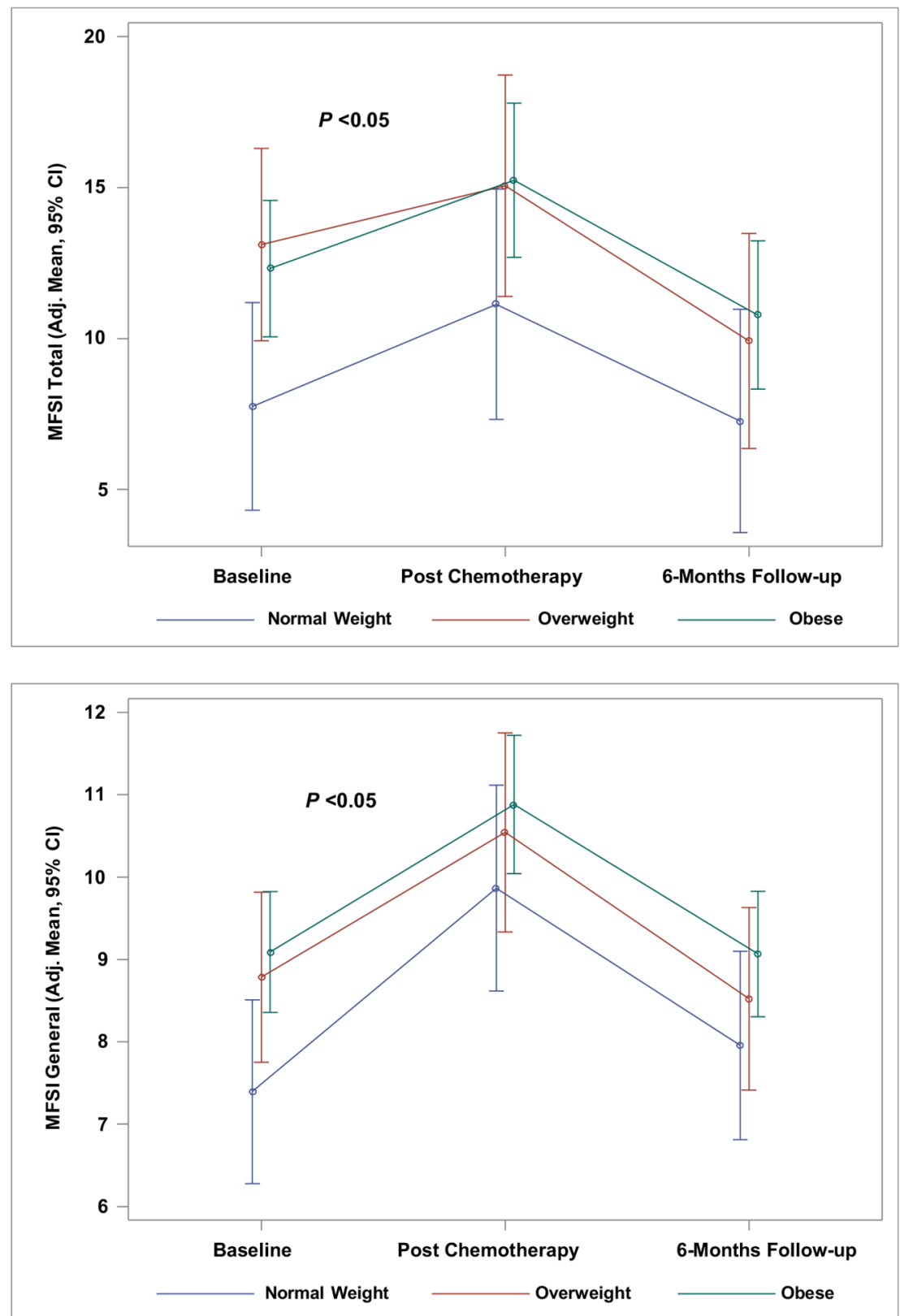

Fig. 2.

LMM analysis: overall MFSI total and MFSI general scores among groups

Means adjusted for age, menopausal status, race, KPS, cancer stage, radiation and exercise status.

$P$-value depicts significant changes between time points for all three groups. 





Inglis et al.

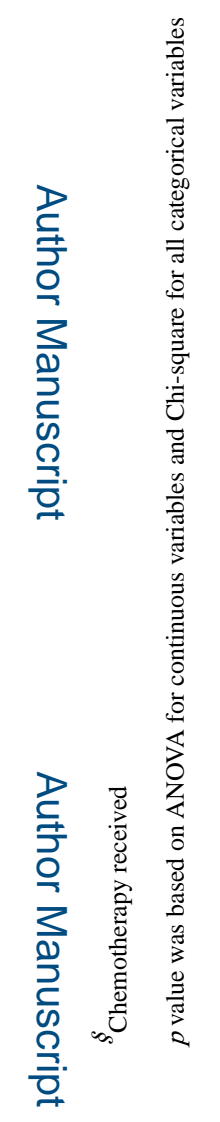

롤

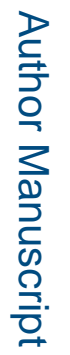

Support Care Cancer. Author manuscript; available in PMC 2020 May 22. 


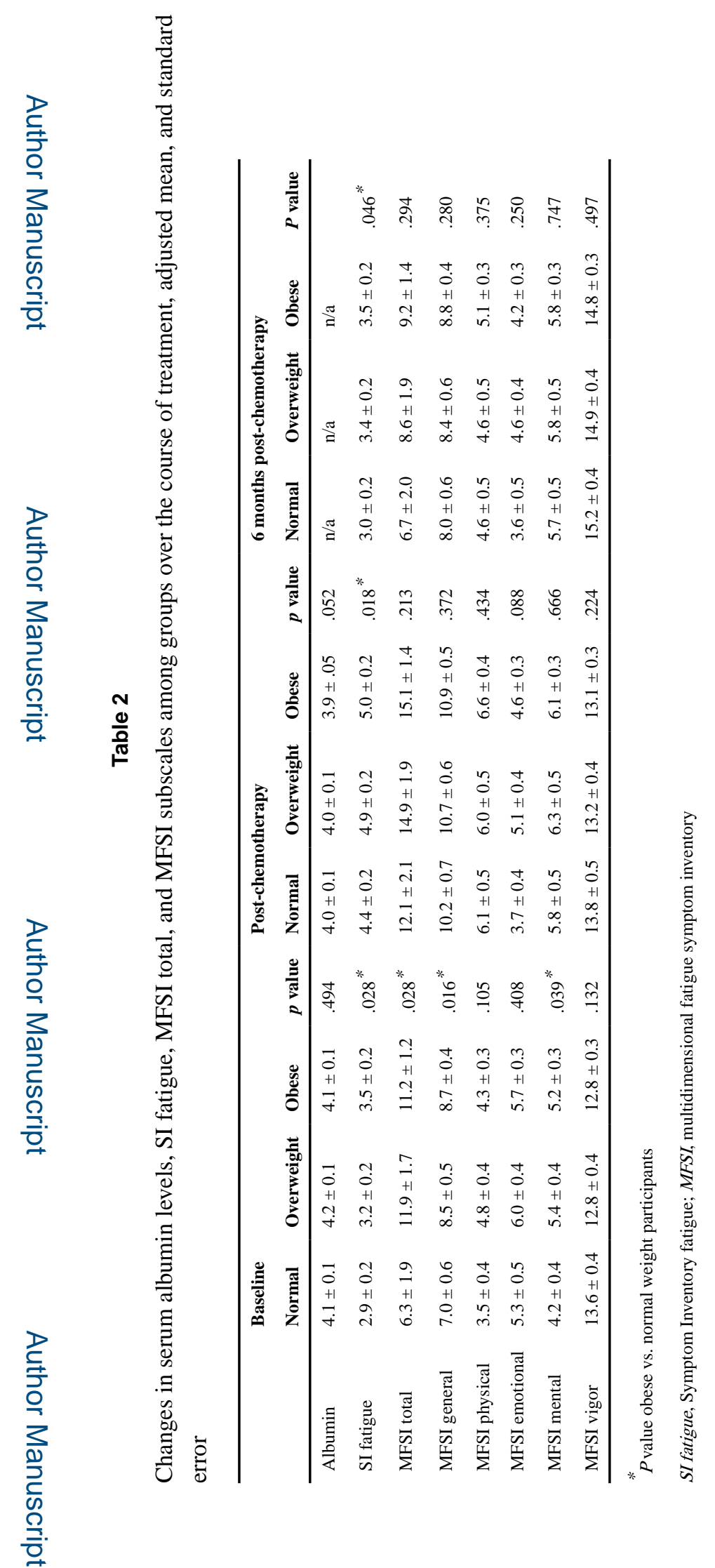

Support Care Cancer. Author manuscript; available in PMC 2020 May 22. 